\title{
Force Field Parameters for 3-Nitrotyrosine and 6-Nitrotryptophan
}

\author{
Yoochan Myung and Sanghwa Han* \\ Department of Biochemistry and Institute for Life Sciences, Kangwon National University, Chunchon 200-701, Korea \\ *E-mail:hansh@kangwon.ac.kr \\ Received July 2, 2010, Accepted July 26, 2010
}

\begin{abstract}
Nitration of tyrosine and tryptophan residues is common in cells under nitrative stress. However, physiological consequences of protein nitration are not well characterized on a molecular level due to limited availability of the 3D structures of nitrated proteins. Molecular dynamics (MD) simulation can be an alternative tool to probe the structural perturbations induced by nitration. In this study we developed molecular mechanics parameters for 3-nitrotyrosine (NIY) and 6-nitrotryptophan (NIW) that are compatible with the AMBER-99 force field. Partial atomic charges were derived by using a multi-conformational restrained electrostatic potential (RESP) methodology that included the geometry optimized structures of both $\alpha$ - and $\beta$-conformers of a capped tripeptide ACE-NIY-NME or ACE-NIW-NME. Force constants for bonds and angles were adopted from the generalized AMBER force field. Torsional force constants for the proper dihedral C-C-N-O and improper dihedral C-O-N-O of the nitro group in NIY were determined by fitting the torsional energy profiles obtained from quantum mechanical (QM) geometry optimization with those from molecular mechanical (MM) energy minimization. Force field parameters obtained for NIY were transferable to NIW so that they reproduced the QM torsional energy profiles of ACE-NIW-NME accurately. Moreover, the QM optimized structures of the tripeptides containing NIY and NIW were almost identical to the corresponding structures obtained from MM energy minimization, attesting the validity of the current parameter set. Molecular dynamics simulations of thioredoxin nitrated at the single tyrosine and tryptophan yielded well-behaved trajectories suggesting that the parameters are suitable for molecular dynamics simulations of a nitrated protein.
\end{abstract}

Key Words: Force field, Nitrotyrosine, Nitrotryptophan, Molecular mechanics, Molecular dynamics simulation

\section{Introduction}

Nitrogen monoxide (nitric oxide) is produced in many cells under physiological and pathological conditions. It is oxidized to nitrite, which is utilized for quantitation of intracellular nitrogen monoxide by the Griess reaction. Nitrite can be oxidized further to nitrogen dioxide radical by metal ion- or heme-catalyzed oxidation. In addition nitrogen monoxide reacts with superoxide anion radical to generate peroxynitrite. Both nitrogen dioxide $^{1}$ and peroxynitrite ${ }^{2}$ are strong nitrating agents which frequently attack tyrosine and tryptophan residues in proteins producing 3-nitrotyrosine ${ }^{3}$ and isomers of singly nitrated tryptophan. ${ }^{4}$

Recent reviews ${ }^{3,5}$ summarized the influences of protein tyrosine nitration on proteolytic degradation, tyrosine phosphorylation, immunogenecity, and mitochondrial physiology. Tyrosine nitration is also implicated in many diseases although it is not clear whether the former is a cause for the latter. ${ }^{5}$ Despite the accumulated evidence for protein nitration, its biological consequences are not well understood on a molecular level primarily due to scarcity of the 3D structures of nitrated proteins. So far crystal structures have been solved only for a few proteins with a nitrated tyrosine ${ }^{6-9}$ and none with a nitrated tryptophan. To overcome the limited structural information on nitrated proteins, we employed a computational approach to probe the structural perturbations induced by protein nitration.

Molecular dynamics (MD) simulation is a method of choice to monitor a protein structure in motion. An MD software equipped with an appropriate force field (the popular AMBER-99, for example) calculates the trajectory of each atom in a protein as a function of time. Usually an MD software package provides force field parameters only for standard residues. To run an MD simulation of a protein containing a modified residue like 3-nitrotyrosine (NIY) or 6-nitrotryptophan (NIW), one must estimate the missing force field parameters: partial atomic charges calculated by a protocol used in the original development of a given force field (RESP charges for the AMBER-99 force field, for example), force constants of the missing internal coordinates, and van der Waals parameters of newly defined atom types. These new parameters must be validated by comparing the structure obtained from quantum mechanical (QM) geometry optimization with that from molecular mechanical (MM) energy minimization.

In an effort to develop a force field appropriate for amino acid residues modified by reactive oxygen and nitrogen species, we previously reported molecular mechanics parameters for $S$ nitrosocysteine that are compatible with AMBER-99. ${ }^{10}$ Adopting a similar approach we here extended the work to NIY and NIW. With the new parameter set, MM calculations accurately reproduced the QM optimized structures of the tripeptides ACENIY-NME and ACE-NIW-NME. The parameters were also proven suitable for MD simulations of a nitrated protein fulfilling our primary goal of expanding the repertoire of modified residues in AMBER-99.

\section{Methods}

Softwares. Chimera ${ }^{11}$ was used to set up the initial structures 
(a)

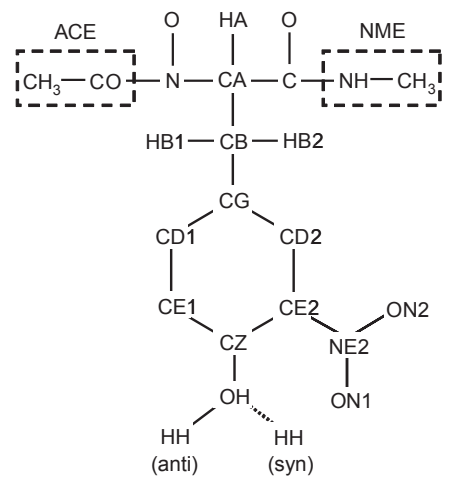

(b)

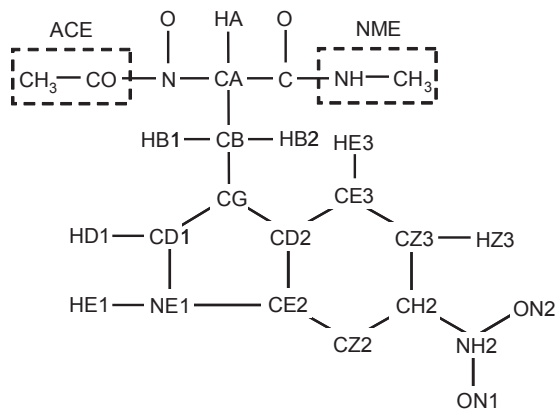

Figure 1. Capped tripeptides containing (a) 3-nitrotyrosine and (b) 6nitrotryptophan. Atom names are given according to the conventions of ffamber99, the AMBER-99 force field in GROMACS format. Also shown are the positions of $\mathrm{HH}$ in anti- and syn-conformations of the hydroxyl group.

of ACE-NIY-NME, ACE-NIW-NME, and nitrated proteins. It was also employed in comparison and RMSD calculation of two structures. Gaussian 03 Rev. E. $01^{12}$ was used in all quantum mechanics (QM) calculations. We carried out geometry optimization at the level of $\mathrm{HF} / 6-31 \mathrm{G}^{*}$ and single-point energy calculations of the optimized structures at the level of B3LYP/ cc-pVTZ. A molecular dynamics (MD) software GROMACS 4.0.5 $5^{13}$ was employed in all molecular mechanics (MM) calculations. The AMBER force field parameters were translated into the GROMACS format as instructed by Sorin and Pande. ${ }^{14}$ Topologies and coordinates obtained from the ANTECHAMBER module of AMBER 9.0 MD software package ${ }^{15}$ were converted to those in GROMACS formats by using a perl script amb2gmx.pl. ${ }^{16}$ Atomic charges were calculated by using the RESP module of AMBER with the help of R.E.D.-III tools. ${ }^{17}$

Calculation of partial atomic charges. To be compatible with the AMBER-99 force field, ${ }^{18}$ partial atomic charges were derived according to a multi-conformational restrained electrostatic potential (RESP) approach. ${ }^{19}$ Both $\alpha$ - and $\beta$-conformers of a tripeptide ACE-NIY-NME or ACE-NIW-NME were included in the charge calculation. Unlike the unmodified residues, however, the number of nitrated proteins with a known 3-D structure is too small to deduce a preferred conformation. Therefore, for the initial structures of tripeptides containing NIY and NIW, we therefore adopted the conformations of unmodified residues that were used in the original AMBER force field development. ${ }^{19}$ The peptide dihedrals $\phi / \psi$ were $-60 /-40$ degrees for the
Table 1. Newly introduced force constants for the nitro group ${ }^{a}$

\begin{tabular}{|c|c|c|c|c|}
\hline Bonds $^{b}$ & $K_{r}^{d}$ & $r_{\mathrm{eq}}^{e}$ & & \\
\hline CA-NO & 322.6 & $1.468(1.45)$ & & \\
\hline NO-O2 & 761.2 & 1.219 (1.19) & & \\
\hline Angles $^{b}$ & $K_{\theta}{ }^{d}$ & $\theta_{\mathrm{eq}}{ }^{e}$ & & \\
\hline CA-CA-NO & 66.9 & $119.54(119.45)$ & & \\
\hline CA-NO-O2 & 68.7 & $118.10(117.76)$ & & \\
\hline $\mathrm{O} 2-\mathrm{NO}-\mathrm{O} 2$ & 76.4 & $127.55(124.47)$ & & \\
\hline Improper torsions ${ }^{c}$ & $V_{n} / 2^{f}$ & $\gamma^{g}$ & $n^{h}$ & \\
\hline $\mathrm{CA}-\mathrm{O} 2-\mathrm{NO}-\mathrm{O} 2$ & 7.28 & 180.0 & 2 & \\
\hline Torsions $^{c}$ & no. of paths ${ }^{i}$ & $V_{n} / 2$ & $\gamma$ & $n$ \\
\hline CA-CA-NO-O2 & 4 & 3.68 & 180.0 & 2 \\
\hline \multicolumn{5}{|c|}{${ }^{a}$ AMBER-99 force field is based on the following energy function. } \\
\hline
\end{tabular}

${ }^{b}$ Values adopted from the generalized AMBER force field. ${ }^{c}$ Newly parametrized in this work. ${ }^{d}$ Force constant for bonds in $\mathrm{kcal} / \mathrm{mol} \cdot \AA^{2}$ or for angles in $\mathrm{kcal} / \mathrm{mol} \cdot \mathrm{rad}^{2}$. ${ }^{e}$ Equilibrium distance in $\AA$ or equilibrium angle in degrees. Numbers in parentheses are the average values obtained from QM optimized structures. ${ }^{f}$ Torsional potential in $\mathrm{kcal} / \mathrm{mol} .{ }^{g}$ Phase offset in degrees. ${ }^{h}$ Periodicity of the torsion. ${ }^{\prime}$ Number of bond paths that the total $V_{n} / 2$ must be divided by. The middle two atoms $\mathrm{CA}$ and $\mathrm{NO}$ are bonded to two CA's and two O2's, respectively, so that the number of bond paths is equal to $2 \times 2$.

$\alpha$-conformer of NIY ( $\alpha$-NIY) and-143/160 for the $\beta$-conformer $(\beta-N I Y)$. For NIW the corresponding values were $-60 /-40$ for the $\alpha$-conformer ( $\alpha$-NIW) and $-144 / 161$ for the $\beta$-conformer ( $\beta$ NIW). The nitro group was placed in plane with the aromatic ring. To comply with the general practice in the RESP procedure, the dihedral CE1-CZ-OH-HH of the hydroxyl group in NIY was set to zero (i.e. anti-conformation) to avoid hydrogen bonding with the nitro group (see Fig. 1). Initial conformations of ACE-NIY-NME and ACE-NIW-NME are summarized in Table S1.

Starting structures of the $\alpha$ - and $\beta$-conformers of ACE-NIYNME or ACE-NIW-NME were subjected to geometry optimization at the level of $\mathrm{HF} / 6-31 \mathrm{G}^{*}$ with $\phi$ and $\psi$ fixed at the initial values. The other internal coordinates were allowed to vary during optimization. Conformations resulting from geometry optimization are also given in Table S1. RESP charges were calculated by using the program RESP implemented in the AMBER 9.0 package. In order to obtain the RESP charges of the central fragment, total charges of ACE and NME were restrained to zero and charges of the peptide bond atoms $\mathrm{C}, \mathrm{O}$, $\mathrm{N}$, and $\mathrm{H}$ were set, respectively, to $0.5973,-0.5679,-0.4157$, and 0.2719 as in the AMBER-99 force field. ${ }^{17}$

Determination of missing force field parameters. As none of the residues listed in AMBER-99 have a nitro group, we need to assign atom types to its nitrogen and oxygen. Based on chemical analogy we assigned a pre-existing atom type $\mathrm{O} 2$ (carboxyl) to the oxygen and a new type NO to the nitrogen. To 
keep the number of new parameters minimal, we assigned the original atom types to $\mathrm{CE} 2$ and $\mathrm{CH} 2$ which now form a new bond with the nitro group. The atom type of $\mathrm{CZ}$ bonded to the hydroxyl group was changed from $\mathrm{C}$ in the unmodified tyrosine to CA in NIY. The van der Waals parameters for the new atom type NO were obtained from the generalized AMBER force field (GAFF) ${ }^{20,21}$ implemented in the ANTECHAMBER module of AMBER 9.0 package.

For the nitro group attached to tyrosine or tryptophan we introduced seven new force constants: two for bonds (CA-NO and $\mathrm{NO}-\mathrm{O} 2$ ), three for angles (CA-CA-NO, CA-NO-O2, and $\mathrm{O} 2-\mathrm{NO}-\mathrm{O} 2$ ), and two for torsions (a proper dihedral CA-CA$\mathrm{NO}-\mathrm{O} 2$ and an improper dihedral CA-NO-O2-O2). Force constants for the bonds and angles were readily obtained from GAFF (see Table 1). As the first step for the derivation of torsional force constants, conformers of $\beta$-NIY were generated by varying the proper dihedral angle CZ-CE2-NE2-ON1 $\left(\tau_{\mathrm{CCNO}}\right)$ from 0 to 180 degrees and the improper dihedral angle CE2-ON1NE2-ON2 $\left(\tau_{\text {CONO }}\right)$ from 180 to 130 degrees. These structures were optimized at the level of $\mathrm{HF} / 6-31 \mathrm{G}^{*}$ with $\phi, \psi$, and $\tau$ fixed at the initial values. Torsional energy profiles were obtained by performing single point energy calculations at the level of B3LYP/cc-pVTZ on the optimized structures. Torsional force constants were determined by fitting the QM torsional energy profiles with the corresponding energies obtained from MM calculations. For the latter we minimized the energy of a conformer by a conjugate gradient method using GROMACS. We changed the torsional force constant until the MM energies reproduced the QM energies. The force constant for $\tau_{\mathrm{CONO}}$ was determined first because its MM torsional energy profile was not sensitive to the force constant for $\tau_{\mathrm{CCNO}}$.

Validation of the force field parameters. To validate the new force field parameters, we compared the QM geometry optimized structures of tripeptides containing NIY and NIW with the corresponding structures obtained from MM energy minimizations in vacuo. Same starting structures were used for both QM and MM calculations. The $\phi$ and $\psi$ angles were frozen at the initial values whereas all other internal coordinates were allowed to vary to reach an energy minimum. Chimera was used to compare a QM geometry optimized structure with the corresponding MM energy minimized structure and to calculate their RMSD value.

Molecular dynamics simulations of nitrated proteins. We nitrated the single tyrosine or the single tryptophan residue in thioredoxin (1 eru.pdb) ${ }^{22}$ by using a structure building tool of Chimera. Molecular dynamics (MD) simulations were performed by using GROMACS with the AMBER-99 force field parameters. An initial structure was immersed in a periodic TIP3P water box of cubic shape whose edge was placed at $10 \AA$ from the protein. The system was electrically neutralized by adding five $\mathrm{Na}^{+}$ions. Particle mesh Ewald method ${ }^{23}$ was used in the calculation of electrostatic energy. Cutoff distances for the Coulomb and van der Waals interactions were 9 and $14 \AA$, respectively. After a short energy minimization step using a conjugate gradient method, the system was subjected to equilibration at $300 \mathrm{~K}$ and 1 bar for $150 \mathrm{ps}$ under the conditions of position restraints for heavy atoms and LINCS constraints ${ }^{24}$ for all bonds. The system was weakly coupled to the external bath by the
Berendsen pressure and temperature coupling. ${ }^{25}$ Finally the position restraints were removed in the production MD calculations keeping all the other conditions unaltered. The results were analyzed using the standard softwares included in the GROMACS package.

\section{Results and Discussion}

Protein tyrosine nitration is common in cells that produce nitrogen monoxide. It often results in loss of protein function presumably due to the structural alteration induced by nitration of tyrosine and tryptophan. Crystal structures are available only for a few proteins with nitrated tyrosine residues, ${ }^{6-9}$ however, so that molecular dynamics (MD) simulation is a good choice for an alternative structural probe. Quality of an MD simulation depends heavily on the force field parameters used. Therefore it is prerequisite for a successful simulation of a nitrated protein to have a good set of parameters including partial atomic charges, van der Waals parameters, and the force constants associated with the nitro group. We developed a force field that is compatible with AMBER-99 because it is a popular force field that can be used in conjunction with the generalized AMBER force field (GAFF), ${ }^{20,21}$ a versatile force field for small molecules.

Geometry optimized structures. Initial structures of ACENIY-NME and ACE-NIW-NME were optimized at the level of $\mathrm{HF} / 6-31 \mathrm{G}^{*}$ and their energies were calculated at the level of B3LYP/cc-pVTZ. After geometry optimization with fixed $\phi$ and $\psi, \alpha$-conformer of ACE-NIY-NME ( $\alpha$-NIY) changed its conformation significantly whereas $\beta$-NIY had similar $\chi$ 's as the initial structure (see Table S1). The nitro group in $\alpha$-NIY and $\beta$-NIY rotated about the $\mathrm{C}-\mathrm{NO}_{2}$ bond by 27.4 and -28.2 degrees, respectively. In contrast, both $\alpha$-NIW and $\beta$-NIW retained their initial structures including the nitro group that remained in plane with the aromatic ring. Energy of the synconformer (see Fig. 1) was lower than that of the anti-conformer by 10.1 and $10.3 \mathrm{kcal} / \mathrm{mol}$ for $\alpha-\mathrm{NIY}$ and $\beta$-NIY, respectively. This corresponds approximately to the hydrogen bond energy of NIY. For comparison we obtained a value of $10.7 \mathrm{kcal} / \mathrm{mol}$ for 2-nitrophenol that agrees reasonably well with the reported value of $12.0 \mathrm{kcal} / \mathrm{mol}^{26}$

Partial atomic charges of 3-nitrotyrosine and 6-nitrotryptophan. In keeping with a minimalist strategy, the atom type of $\mathrm{O}$ in the nitro group was assigned by analogy to $\mathrm{O} 2$, which is already included in AMBER-99. A new atom type NO was assigned to the $\mathrm{N}$ atom and its van der Waals parameters were obtained from GAFF. In fact the van der Waals parameters of NO were the same as those of the other nitrogen atom types in AMBER-99.

To comply with the original protocol for the AMBER-99 force field, a restrained electrostatic potential (RESP) appro$\mathrm{ach}^{18,19}$ was used in the calculation of partial atomic charges. Since hydrogen bonding should be avoided in an RESP charge derivation, the hydroxyl group of NIY was placed at the opposite side of the nitro group (anti-conformation; see Fig. 1). Both $\alpha$ - and $\beta$-conformers after geometry optimization were included in the calculation. As summarized in Table S2, the partial atomic charge of $\mathrm{N}$ in the nitro group had a large positive value $(+0.79$ 
Table 2. Comparison of the structures obtained from quantum mechanical geometry optimizations and molecular mechanical energy minimizations

\begin{tabular}{|c|c|c|c|c|c|c|c|c|}
\hline & \multicolumn{2}{|c|}{$\alpha-\mathrm{NIY}^{a}$} & \multicolumn{2}{|c|}{$\beta-\mathrm{NIY}^{a}$} & \multicolumn{2}{|c|}{$\alpha-\mathrm{NIW}^{a}$} & \multicolumn{2}{|c|}{$\beta-\mathrm{NIW}^{a}$} \\
\hline & $\mathrm{QM}$ & MM & QM & MM & $\mathrm{QM}$ & MM & QM & MM \\
\hline$\tau_{\mathrm{CCOH}}^{b}$ & 0.7 & 3.9 & 0.5 & -3.6 & - & - & - & - \\
\hline$\tau_{\mathrm{CCNO}}{ }^{c}$ & 27.4 & 28.3 & -28.2 & -26.4 & 0.1 & -0.1 & -0.2 & -0.1 \\
\hline$\tau_{\mathrm{CONO}}{ }^{d}$ & -178.7 & -178.2 & 178.9 & 178.8 & 179.9 & 179.9 & -179.9 & -179.8 \\
\hline
\end{tabular}

${ }^{a} \alpha$ - and $\beta$-XXX mean a tripeptide ACE-XXX-NME in $\alpha$ - and $\beta$-conformation, respectively. ${ }^{b}$ Proper dihedral CE1-CZ-OH-HH. ${ }^{c}$ Proper dihedral CZ-CE2- NE2-ON1 for NIY or CZ2-CH2-NH2-ON1 for NIW. ${ }^{d}$ Improper dihedral CE2-ON1-NE2-ON2 for NIY or CH2-ON1-NE2-ON2 for NIW.

for NIY and +0.75 for NIW) whereas O had a large negative value ( -0.46 for both NIY and NIW). Compared with the unmodified residues a large change was observed at the carbon atoms bonded to the nitro group, i.e. CE2 of NIY and $\mathrm{CH} 2$ of NIW, that lost electron density upon nitration. The next closest atoms became more negative in NIY (CD2 and CZ) but more positive in NIW (CZ2 and CZ3). To assess the polarity around the nitrated residues we calculated the dipole moments of phenol, indole, and their nitro derivatives. Dipole moment of 5.07 and 2.05 D were obtained for phenol and indole, respectively. Upon nitration the dipole moment increased to 6.39 D for nitrophenol in anti-conformation but decreased to $3.98 \mathrm{D}$ for nitrophenol in syn-conformation. The results are consistent with the reported values. ${ }^{26-27}$ Since the hydrogen-bonded conformation of NIY is prevalent in proteins, the local polarity is likely to decrease when a tyrosine residue is nitrated. Dipole moment of nitroindole was $6.62 \mathrm{D}$, more than three times as large as that of unmodified indole. Nitration of a tryptophan residue would increase local polarity.

Detemination of the force constants associated with the nitro group. We need to add two new force constants for bonds (CA$\mathrm{NO}$ and $\mathrm{NO}-\mathrm{O} 2$ ) and three for angles (CA-CA-NO, CA-NO$\mathrm{O} 2$, and $\mathrm{O} 2-\mathrm{NO}-\mathrm{O} 2$ ) associated with the nitro group in NIY and NIW. The atom type of CZ was changed from $\mathrm{C}$ in tyrosine to $\mathrm{CA}$ in NIY to reduce the number of force constants. In favor of our assignment, $\mathrm{CZ}$ is also defined as $\mathrm{CA}$ in GAFF. These force constants for bonds and angles can be readily obtained from $\mathrm{GAFF}^{20,21}$ by running the ANTECHAMBER module of AMBER and the results are summarized in Table 1. Equilibrium bond lengths and angles agreed well with those from QM optimized structures. We therefore used the GAFF parameters for bonds and angles without modifications.

We next turned to torsional force constants. Since torsional degrees of freedom are an important determinant of the protein conformation, we need to estimate their force constants very accurately. X-ray structures of proteins containing NIY residues show a rotation about the $\mathrm{C}-\mathrm{NO}_{2}$ bond ${ }^{6,7,9}$ as well as an outof-plane deformation ${ }^{6}$ of the nitro group. Therefore we decided to obtain refined force constants for both the proper dihedral CA-CA-NO-O2 ( $\left.\tau_{\mathrm{CCNO}}\right)$ and improper dihedral CA- O2- NO-O2 $\left(\tau_{\mathrm{CONO}}\right)$. Preliminary MM calculations showed that the $\tau_{\mathrm{CONO}}$ torsional energy is insensitive to the $\tau_{\mathrm{CCNO}}$ force constant so that the $\tau_{\mathrm{CONO}}$ force constant was determined first.

As an experimental profile for the $\tau_{\mathrm{CONO}}$ torsion was not available, we calculated it using an $a b$ initio method. A series of structures of $\beta$-NIY were generated by varying $\tau_{\mathrm{CONO}}$ at an

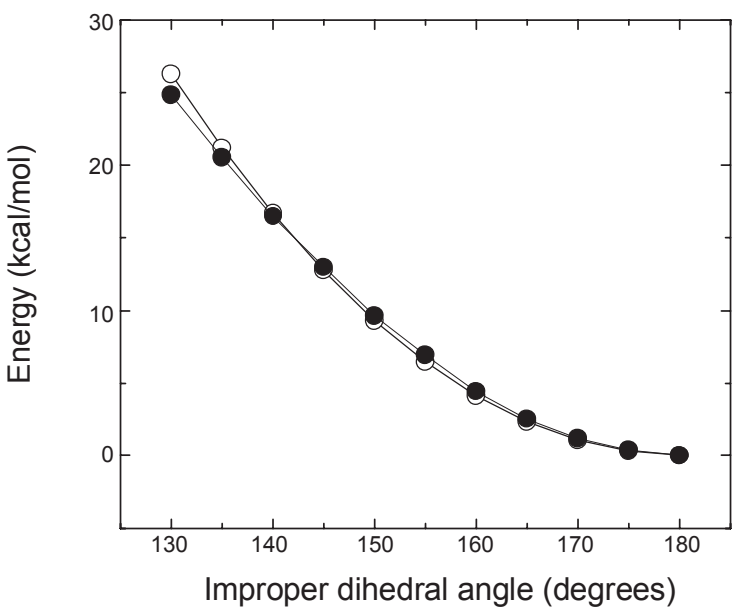

Figure 2. Quantum mechanical (closed circles) and molecular mechanical (open circles) energy profiles of 3-nitrotyrosine in $\beta$-conformation as a function of the improper dihedral angle $\tau_{\mathrm{CONO}}$. The $\mathrm{QM}$ energies were calculated at the level of B3LYP/6-31G*//HF/6-31G* and the MM energies were calculated with the force constants $\left(V_{n} / 2\right) 7.28$ and $3.68 \mathrm{kcal} / \mathrm{mol}$ for $\tau_{\mathrm{CONO}}$ and $\tau_{\mathrm{CCNO}}$, respectively.

interval of 5 degrees. Their energies were calculated at the level of B3LYP/cc-pVTZ//HF/6-31G*. The peptide dihedrals ( $\phi$ and $\psi$ and $\tau_{\mathrm{CONO}}$ were fixed during optimization whereas all other coordinates were allowed to vary. The calculated profile of the $\tau_{\mathrm{CONO}}$ torsion was presented in Fig. 2 (closed circles). The $\tau_{\mathrm{CONO}}$ torsional energy at 150 degrees was $9.30 \mathrm{kcal} / \mathrm{mol}$, which is comparable to a reported value of $11.4 \mathrm{kcal} / \mathrm{mol}$ for nitrobenzene. $^{28}$ The QM torsional energy profile was then fitted with the energies obtained by MM energy minimizations performed on the same structures that were used in the QM optimizations. With a value of $7.28 \mathrm{kcal} / \mathrm{mol}$ for the $\tau_{\text {CONO force constant }}\left(V_{n} / 2\right.$ according to the AMBER convention), we observed a good agreement between the QM (closed circles) and MM energies (open circles). As mentioned above, the improper torsional energy was insensitive to the $\tau_{\mathrm{CCNO}}$ force constant. We therefore fixed the $\tau_{\mathrm{CONO}}$ force constant at $7.28 \mathrm{kcal} / \mathrm{mol}$ in the next step.

To obtain the $\tau_{\mathrm{CCNO}}$ force constant we need to know torsional energies as a function of $\tau_{\mathrm{CCNO}}$. Again we constructed structures $\beta$-NIY by increasing $\tau_{\mathrm{CCNO}}$ at an interval of 10 degrees. Their torsional energies were calculated at the level of B3LYP/cc$\mathrm{pVTZ} / / \mathrm{HF} / 6-31 \mathrm{G}^{*}$ and a barrier height of $2.48 \mathrm{kcal} / \mathrm{mol}$ was obtained for $\beta$-NIY in anti-conformation. As shown in Fig. 3, the QM torsional energies (closed circles) were fitted almost perfectly with the MM energies (open circles) when a value of 


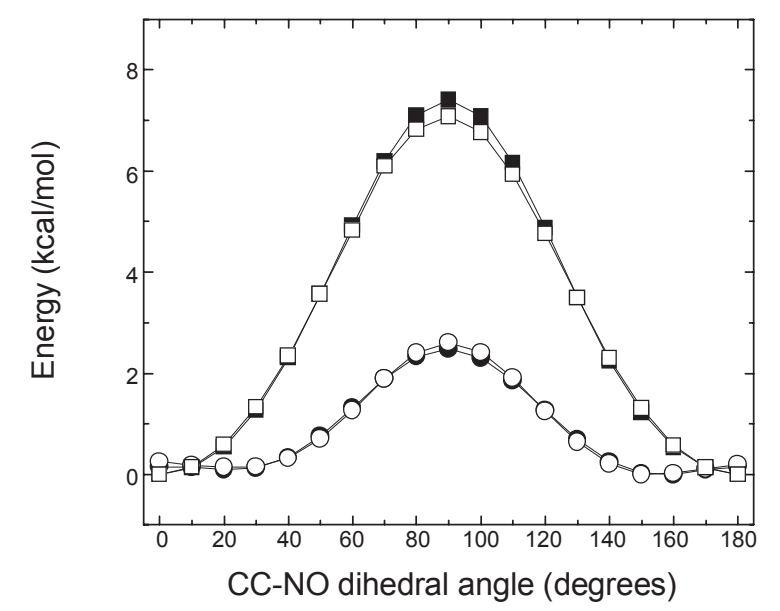

Figure 3. Quantum mechanical (closed) and molecular mechanical (open) torsional energy profiles of the proper dihedral angle $\tau_{\mathrm{CCNO}}$ for 3 -nitrotyrosine (circles) and 6-nitrotryptophan (squares) in $\beta$-conformations. Torsional force constants for 3-nitrotyrosine were used for 6nitrotryptophan without modification. Note the molecular mechanics parameters produce a good fit for both 3-nitrotyrosine and 6-nitrotryptophan.

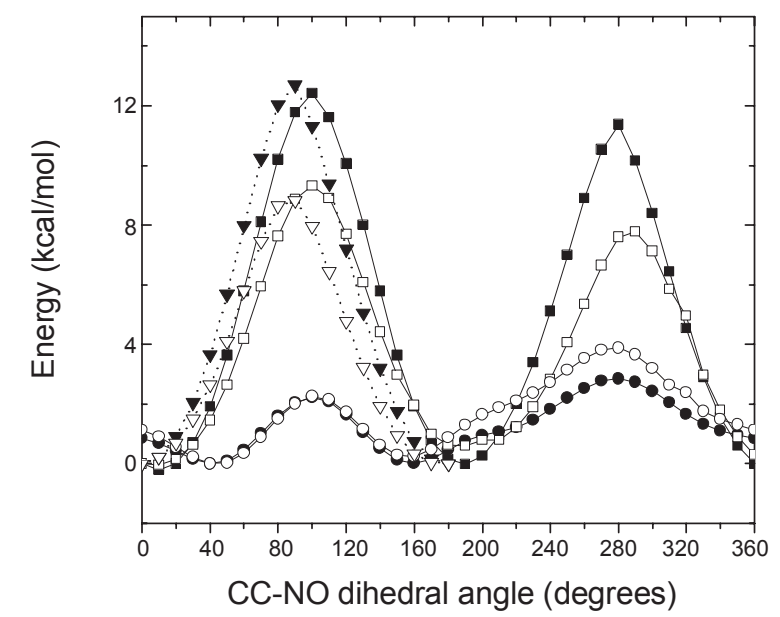

Figure 4. Torsional energy profiles of the proper dihedral $\tau_{\mathrm{CCNO}}$ for 3 nitrotyrosine with an out-of-plane deformation in the nitro group $(\tau$ $\mathrm{CONO}=160$ degrees). Quantum mechanical (closed) and molecular mechanical (open) calculations were performed on anti- (circles) and syn-conformers (squares) of 3-nitrotyrosine in $\beta$-conformation. Also shown are the QM (closed triangles) and $\mathrm{MM}$ (open triangles) torsional energy profiles of undistorted $\left(\tau_{\mathrm{CONO}}=0\right) 3$-nitrotyrosine.

$3.68 \mathrm{kcal} / \mathrm{mol}$ was used for the $\tau_{\mathrm{CCNO}}$ force constant $V_{n} / 2$. We also calculated the $\tau_{\mathrm{CCNO}}$ torsional energies of $\beta$-NIW (closed squares). The torsional barrier of $\tau_{\mathrm{CCNO}}$ in NIW $(7.41 \mathrm{kcal} / \mathrm{mol})$ was almost three times as large as that in NIY. Without changing the force constants obtained for $\beta$-NIY including $\tau_{\mathrm{CONO}}$ and $\tau_{\mathrm{CCNO}}$, the QM torsional energy profile of $\beta$-NIW was almost perfectly reproduced by the MM calculations (open squares). A good quality fitting was also obtained for $\alpha$-NIY and $\alpha$-NIW (not shown). We can conclude that the values 3.68 and 7.28 $\mathrm{kcal} / \mathrm{mol}$ for the improper $\left(\tau_{\mathrm{CONO} O}\right)$ and proper $\left(\tau_{\mathrm{CCNO}}\right)$ torsions, respectively, are transferable to other aromatic nitro compounds.

A value of $4.54 \pm 0.72 \mathrm{kcal} / \mathrm{mol}$ was obtained for the $\tau_{\mathrm{CCNO}}$ torsional barrier of nitrobenzene from gas-phase electron di- ffraction. ${ }^{29}$ Our QM barrier $(2.48 \mathrm{kcal} / \mathrm{mol})$ for NIY in anticonformation, where hydrogen bonding is excluded, was much smaller than the above experimental value. In order to account for the difference we calculated the $\tau_{\mathrm{CCNO}}$ energy barriers for nitrobenzene and anti-nitrophenol at the level of B3LYP/cc$\mathrm{pVTZ} / / \mathrm{HF} / 6-31 \mathrm{G}^{*}$. We observed that hydroxylation of nitrobenzene resulted in a large decrease in the $\tau_{\mathrm{CCNO}}$ torsional barrier (from $5.85 \mathrm{kcal} / \mathrm{mol}$ for nitrobenzene to $1.64 \mathrm{kcal} / \mathrm{mol}$ for antinitrophenol). The value $5.85 \mathrm{kcal} / \mathrm{mol}$ for nitrobenzene was in reasonable agreement with the experimental value of $4.54 \pm$ $0.72 \mathrm{kcal} / \mathrm{mol}^{29}$ and a better agreement was obtained if calculated at a higher level. ${ }^{28}$ To further test the quality of our parameters and see the effect of out-of-plane deformation on the $\tau_{\mathrm{CCNO}}$ torsion, we calculated the $\tau_{\mathrm{CCNO}}$ torsional energies with $\tau_{\mathrm{CONO}}$ fixed at 160 degrees, a large distortion considering the $\tau_{\text {CONO }}$ force constant. Since the two-fold symmetry is lifted, we need to calculate the torsional profile of $\tau_{\mathrm{CCNO}}$ ranging from 0 to 360 degrees. QM torsional profile of $\beta$-NIY in anti-conformation (closed circles) had the shifted maxima at 100 and 280 degrees as shown in Fig. 4. Although deviations were evident in the range between 180 and 360 degrees, the MM energies (open circles) agreed reasonably well with the QM energies. The hydrogen-bonded syn-conformer showed a larger discrepancy between the QM (closed squares) and MM calculations (open squares) than the anti-conformer. This is not surprising considering that the atomic charges were derived for the anticonformation. The $\tau_{\mathrm{CCNO}}$ energy barrier of undistorted $\left(\tau_{\mathrm{CONO}}=\right.$ 0) $\beta$-NIY in syn-conformation was 12.72 and $8.84 \mathrm{kcal} / \mathrm{mol}$ by $\mathrm{QM}$ (closed triangles) and MM calculations (open triangles), respectively. The MM value $(8.84 \mathrm{kcal} / \mathrm{mol})$ was closer than the $\mathrm{QM}$ value to the experimental value of $7.41 \pm 1.20 \mathrm{kcal} / \mathrm{mol}$ obtained for 2-nitrophenol. ${ }^{29}$ Nevertheless the overall characteristics of the QM torsional profiles were reproduced reasonably well by the MM calculations even for the structures with a large out-of-plane deformation.

Validation of the force field parameters. To test the validity of our parameters, we compared the structures obtained from QM geometry optimization and MM energy minimization. Initial structures were the same for QM and MM calculations. The conformations were adopted from the corresponding unmodified residues employed in the AMBER-99 force field development (see Table S1). The nitro group was in plane with the aromatic ring and the hydroxyl group in NIY had an anticonformation to avoid hydrogen bonding. Only the peptide dihedrals $\phi$ and $\psi$ were kept constant and all other coordinates were allowed to vary freely until during QM and MM calculations. As shown in Fig. 5, QM geometry optimization and MM energy minimization on the NIY-containing tripeptides produced almost identical structures attesting the good quality of our parameters. The RMSD's calculated for the coordinates of the heavy atoms in the QM and MM optimized structures were 0.092 and $0.096 \AA$ for $\alpha$-NIY and $\beta$-NIY, respectively. A good agreement was also observed for $\beta$-NIW (RMSD 0.111 $\AA$ ). In $\alpha$-NIW, however, the discrepancy was significant (RMSD $0.292 \AA$ ). If one considers only the nitrophenol and nitroindole moieties, however, the agreement between the QM and MM optimized structures was almost perfect for all four structures as summarized in Table 2. Tilting of the hydroxyl group was 
(a)

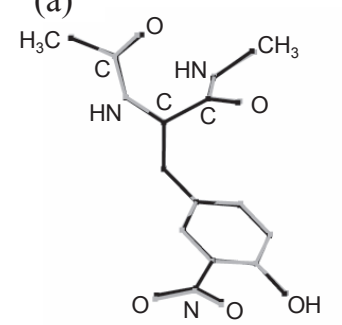

(c)

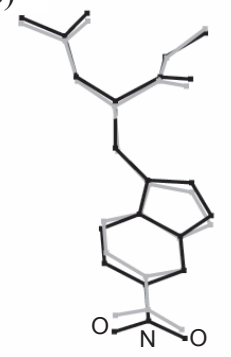

(b)<smiles>CC(O)NC(CC1CCC(O)C(C(=O)[O-])C1)C(C)O</smiles>

(d)

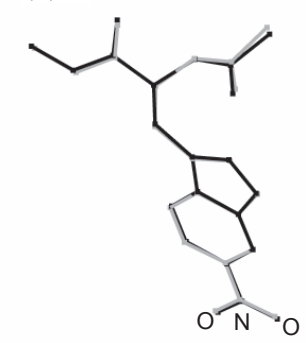

Figure 5. Comparison of the structures obtained from quantum mechanical geometry optimizations (dark) and molecular mechanical energy minimizations (light) of capped tripeptides of 3 -nitrotyrosine in (a) $\alpha$ and (b) $\beta$-conformations and 6-nitrotryptophan in (c) $\alpha$ - and (d) $\beta$ conformations. Only heavy atoms are shown and included in the RMSD calculations

(a)

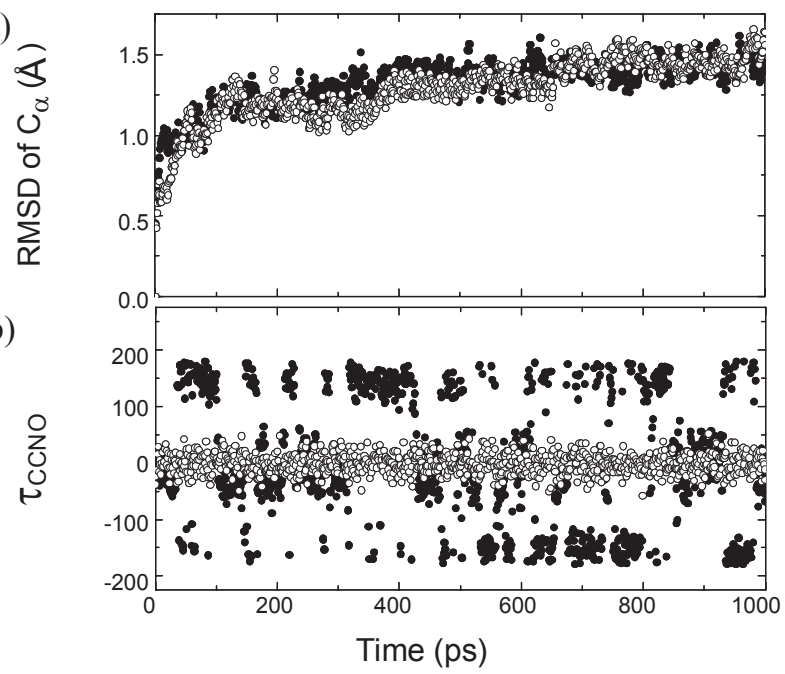

Figure 6. Trajectories of (a) $\mathrm{C}_{\alpha}$ RMSD and (b) proper dihedral $\tau_{\mathrm{CCNO}}$ obtained from a $1 \mathrm{~ns}$ molecular dynamics simulation of thioredoxin nitrated at $\operatorname{Tyr}^{49}$ (closed) and $\operatorname{Trp}^{31}$ (open).

negligible in both QM and MM optimized structures. Rotation of the nitro group was remarkable, however. The nitro group was in plane with the aromatic ring $\left(\tau_{\mathrm{CCNO}}=0\right)$ in the initial structures. After a QM optimization it rotated about the C-NO bond by 27.4 and -28.2 degrees for $\alpha-\mathrm{NIY}$ and $\beta$-NIY, respectively. The corresponding values from MM calculations, 28.3 and -26.4 degrees, demonstrate that the rotation of the nitro group was accurately reproduced by MM calculations in both size and direction. In NIW the nitro group stayed in plane with the indole ring during both QM and MM optimizations. The improper dihedral of the nitro group $\tau_{\mathrm{CONO}}$ also showed a good agreement between QM and MM calculations. Overall the parameters developed in this study were good enough to reproduce the QM optimized structures of ACE-NIY-NME and ACENIW-NME.

Molecular dynamics simulations of nitrated proteins. So far crystal structures have been solved only for a few proteins with a nitrotyrosine, i.e., $\mathrm{Cu}, \mathrm{Zn}$-superoxide dismutase $(\mathrm{CuZn}$ SOD), ${ }^{6} \mathrm{MnSOD},{ }^{7}$ glutathione reductase, ${ }^{8}$ and laccase. ${ }^{9}$ Structures of nitrotryptophan-containing proteins are not available yet. We were not able to carry out MD simulations on these proteins, however, since some parameters are not provided in the AMBER-99 force field (for example, deprotonated histidine anion in $\mathrm{CuZnSOD}, \mathrm{Mn}$ in MnSOD, FAD in glutathione reductase, and glycosylation in laccase). Therefore we chose thioredoxin (Trx) as a model protein on which we previously reported MD simulations. ${ }^{10,30} \operatorname{Trx}$ is a small molecule with a single tyrosine and tryptophan residue on the surface that are exposed to incoming nitrating agents. In fact Trx is known to be nitrated at tyrosine. ${ }^{31,32}$ Nitration of its tryptophan has not been reported.

Initial structures of Trx (1eru.pdb) nitrated at $\operatorname{Tyr}^{49}$ and $\operatorname{Trp}^{31}$ were constructed by introducing a nitro group at $\mathrm{CE} 2$ and $\mathrm{CH} 2$, respectively, with the help of a structure building tool of Chimera. The nitro group was always in plane with the aromatic ring in the starting structures. Each protein was immersed in water and subjected sequentially to a short energy minimization, a position-restrained equilibration, and a $1 \mathrm{~ns}$ production MD simulation. Fig. 6a shows the RMSD's of $\mathrm{C}_{\alpha}$ of Trx with a nitrotyrosine (closed circles) and a nitrotryptophan (open circles). We obtained a well-behaved trajectory that reached equilibrium at an early time and stayed stable thereafter. This suggests that our new parameters are suitable for an MD simulation of a nitrated protein.

We next examined the rotational motion of the nitro group. As shown in Fig. 6 b (closed circles), the in-plane nitro group of NIY in anti-conformation flipped back and forth, and $\tau_{\mathrm{CCNO}}$ spanned angles in between. A low torsional energy barrier (2.61 kcal/mol) could be responsible for the relatively easy rotation. Flipping was nearly impossible for NIY in syn-conformation so that the nitro group stayed in the same orientation most of the time (not shown). This could be due to hydrogen bonding in syn-conformation that resulted in a high rotation barrier $(8.84 \mathrm{kcal} / \mathrm{mol})$. However, though infrequent, the $\mathrm{C}-\mathrm{NO}_{2}$ bond could rotate as much as 70 degrees. Crystal structures of proteins with NIY agree with our prediction. Distribution of the $\tau_{\mathrm{CCNO}}$ angle showed a large dispersion and the maximum value of 69.8 degrees was found in nitrated CuZnSOD. ${ }^{6}$ The

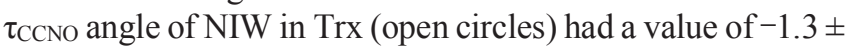
17.5 degrees (Fig. 6b, open circles), demonstrating that the nitro group could not flip over but rotate about the $\mathrm{C}-\mathrm{NO}_{2}$ bond to some extent. This can also be explained in part by the high torsional barrier of the $\tau_{\mathrm{CCNO}}$ in NIW $(7.41 \mathrm{kcal} / \mathrm{mol})$.

Out-of-plane deformation of the nitro group was small in the MD simulations due to its large force constant. The $\tau_{\mathrm{CONO}}$ angle had a value of $180.1 \pm 5.6$ degrees for NIY and $180.7 \pm$ 5.7 degrees for NIW. Among ten tyrosine residues in four nitrated proteins with a known crystal structure, ${ }^{6-9}$ eight did not 
suffer a significant deformation $\left(\tau_{\mathrm{CONO}}=2.4 \pm 3.2\right.$ degrees $)$. Only two NIY's out of four in $\mathrm{CuZnSOD}^{6}$ had unusually large values (26.2 and 30.5 degrees) of $\tau_{\text {CONO }}$. We could not reproduce such large deformations by MD simulations although infrequent deviations as large as 20 degrees were observed.

\section{Conclusions}

We developed AMBER-99 compatible force field parameters for 3-nitrotyrosine and 6-nitrotryptophan. Molecular mechanics calculations accurately reproduced the quantum mechanically optimized structures of capped tripeptides with a nitrated residue, attesting the validity of these parameters. Suitability of the parameters for molecular dynamics simulations of nitrated proteins in general was demonstrated by a well-behaved trajectory observed in the simulations of model thioredoxin with a 3-nitrotyrosine and 6-nitrotryptophan. Equipped with the appropriate force field parameters, molecular dynamics simulations can be an alternative tool for probing the structural perturbations induced by nitration.

Acknowledgments. This study was supported by 200 Research Grant from Kangwon National University.

Supporting Information Available. Conformations of the initial and optimized structures, partial atomic charges of 3-nitrotyrosine and 6-nitrotryptophan, newly introduced force constants for the nitro group in the GROMACS format.

\section{References}

1. Roncone, R.; Barbieri, M.; Monzani, E.; Casella, L. Coord. Chem. Rev. 2006, 250, 1286.

2. Beckman, J. S. Chem. Res. Toxicol. 1996, 9, 836.

3. Abello, N.; Kerstjens, H. A.; Postma, D. S.; Bischoff, R. J. Proteome. Res. 2009, 8, 3222.

4. Yamakura, F.; Ikeda, K. Nitric Oxide 2006, 14, 152.

5. Souza, J. M.; Peluffo, G.; Radi, R. Free Radic. Biol. Med. 2008, $45,357$.

6. Smith, C. D.; Carson, M.; van der Woerd, M.; Chen, J.; Ischiropoulos, H.; Beckman, J. S. Arch. Biochem. Biophys. 1992, 299, 350.

7. Savvides, S. N.; Scheiwein, M.; Bohme, C. C.; Arteel, G. E.; Karplus, P. A.; Becker, K.; Schirmer, R. H. J. Biol. Chem. 2002, 277, 2779.

8. Quint, P.; Reutzel, R.; Mikulski, R.; McKenna, R.; Silverman, D. N. Free Radic. Biol. Med. 2006, 40, 453.
9. Lyashenko, A. V.; Zhukhlistova, N. E.; Gabdoulkhakov, A. G.; Zhukova, Y. N.; Voelter, W.; Zaitsev, V. N.; Bento, I.; Stepanova, E. V.; Kachalova, G. S.; Koroleva, O. V.; Cherkashyn, E. A.; Tishkov, V. I.; Lamzin, V. S.; Schirwitz, K.; Morgunova, E. Y.; Betzel, C.; Lindley, P. F.; Mikhailov, A. M. Acta Crystallogr. Sect. F Struct. Biol. Cryst. Commun. 2006, 62, 954.

10. Han, S. Biochem. Biophys. Res. Commun. 2008, 377, 612.

11. Goddard, T. D.; Huang, C. C.; Ferrin, T. E. J. Struct. Biol. 2007, 157, 281.

14. Frisch, M. J. et al., Gaussian03 program, Revision A.9 package, Gaussian, Inc.; Pittsburgh, PA, 2003.

13. Hess, B.; Kutzner, C.; van der Spoel, D.; Lindahl, E. J. Chem. Theory Comput. 2008, 4, 435.

14. Sorin, E. J.; Pande, V. S. Biophys. J. 2005, 88, 2472. Also visit http://chemistry.csulb.edu/ffamber

15. Pearlman, D. A.; Case, D. A.; Caldwell, J. W.; Ross, W. R.; Cheatham, I. T. E.; De-Bolt, S.; Ferguson, D.; Seibel, G.; Kollman, P. Comp. Phys. Commun. 1995, 91, 1 .

16. Mobley, D. L.; Chodera, J. D.; Dill, K. A. J. Chem. Phys. 2006, 125,084902 .

17. Pigache, A.; Cieplak, P.; Dupradeau, F.-Y. Automatic and highly reproducible RESP and ESP charge derivation: Application to the development of programs RED and X RED, 227th ACS National Meeting, Anaheim, CA, USA, March 28 - April 1, 2004. Also visit $\mathrm{http} / / \mathrm{q} 4 \mathrm{md}-$ forcefieldtools.org/RED/

18. Wang, J.; Cieplak, P.; Kollman, P. A. J. Comp. Chem. 2000, 21, 1049.

19. Cieplak, P.; Cornell, W. D.; Bayly, C.; Kollman, P. A. J. Comput. Chem. 1995, 16, 1357.

20. Wang, J.; Wolf, R. M.; Caldwell, J. W.; Kollman, P. A.; Case, D. A. J. Comput. Chem. 2004, 25, 1157.

21. Wang, J.; Wang, W.; Kollman, P. A.; Case, D. A. J. Mol. Graph. Model. 2006, 25, 247.

22. Weichsel, A.; Gasdaska, J. R.; Powis, G.; Montfort, W. R. Structure 1996, 4, 735.

23. Essmann, U.; Perera, L.; Berkowitz, M. L.; Darden, T.; Lee, H.; Pedersen, L. G. J. Chem. Phys. 1995, 103, 8577.

24. Hess, B.; Bekker, H.; Berendsen, H. J. C.; Fraaije, J. G. E. M. J. Comp. Chem. 1997, 18, 1463.

25. Berendsen, H. J. C.; Postma, J. P. M.; van Gunsteren, W. F.; DiNola, A.; Haak, J. R. J. Chem. Phys. 1984, 81, 3684.

26. Buemi, G. Chem. Phys. 2002, 282, 181.

27. Granzhan, V. A.; Kolesnik, M. I. J. Struct. Chem. 1971, 11, 995.

28. Takezaki, M.; Hirota, N.; Terazima, M.; Sato, H.; Nakajima, T.; Kato, S. J. Phys. Chem. 1997, 101, 5190.

29. Borisenko, K. B.; Hargittai, I. J. Mol. Struct. 1996, 382, 171.

30. Han, S. Biochem. Biophys. Res. Commun. 2007, 362, 532.

31. Tao, L.; Jiao, X.; Gao, E.; Lau, W. B.; Yuan, Y.; Lopez, B.; Christopher, T.; RamachandraRao, S. P.; Williams, W.; Southan, G,; Sharma, K.; Koch, W.; Ma, X. L. Circulation 2006, 114, 1395.

32. Zhang, H.; Tao, L.; Jiao, X.; Gao, E.; Lopez, B. L.; Christopher, T. A.; Koch, W.; Ma, X. L. Free Radic. Biol. Med. 2007, 43, 39. 